Ingo Tobehn-Steinhäuser, Susanne Kersten, Jaqueline Stauffenberg, Heike Wünscher, Christian Zeitnitz, and Thomas Ortlepp

\title{
First measurements with a radiation hard relative humidity sensor for the ATLAS Experiment
}

\begin{abstract}
A radiation hard humidity sensor, based on an inter-digital structure is developed for the upgrade of the Large Hadron Collider (LHC) to high-luminosity LHC (HL-LHC). In this context, the interior of the detector of the ATLAS Experiment will be replaced. To guarantee a failure-free operation over the whole time, it has to be proven that the humidity in the detector region is negligible. To achieve the required very small size of the humidity sensor, double-sided wafer processing is used; furthermore the inter-digital structures consist of 3D fingers to decrease the necessary area. The otherwise customary but not radiation hard polymer as a sensitive layer is replaced by an $\mathrm{SiO}_{2}$ matrix. In order to overcome the great distance between sensor and data processing, very compact signal preprocessing is also necessary.
\end{abstract}

Keywords: capacitive humidity-sensor, radiation hard, low residual moisture

\section{Introduction}

Currently the ATLAS collaboration develops a concept for the new interior detector which is completely made of silicon semi-conductor detectors [1]. ATLAS was originally an acronym for "a toroidal LHC apparatus", but is now only used as a proper name. The innermost area is formed of multiple layers of pixel detectors, further out follow strip detectors. The coolant of $-40^{\circ} \mathrm{C}$ requires an extremely low relative humidity $(\mathrm{RH})$ of the atmosphere in order to avoid condensation on the electronics in all circumstances. Therefore, a permanent monitoring of the $\mathrm{RH}$ and the dew point by determining moisture sensors is essential for the safe operation of the detector. (The dew point of the environment should be $<-40^{\circ} \mathrm{C}$ ). In addition to the measurement of very low amounts of residual moisture at the low temperatures, the radiation hardness of humidity sensors is a further requirement. Assuming that the ATLAS detector will operate on the high-luminosity large hadron collider (HL-LHC) for ten years, one

Ingo Tobehn-Steinhäuser, Jaqueline Stauffenberg, Heike Wünscher, Thomas Ortlepp, CiS Forschungsinstitut für Mikrosensorik GmbH, Erfurt, Germany, e-mail: itobehn@cismst.de Susanne Kersten, Christian Zeitnitz, Fachgruppe Physik; exp. Teilchenphysik, Wuppertal, Germany 
would expect an integrated luminosity of $3000 \mathrm{fb}^{-1}$. Commercially available moisture sensors use polymers as a wet-sensitive layer and are unsuitable for use because of the lack of radiation hardness. Sensors used so far always had to be replaced after a relatively short time because they changed their characteristics [2].

\section{Experimental setup}

\subsection{The ATLAS Experiment}

For the upgrade of the LHC to the HL-LHC, the ATLAS experiment will replace the entire internal detector to handle both the high radiation load and the increased data rates. This is currently planned for the year 2022, but it is also possible that parts of the internal detector have to be exchanged in 2018. The ATLAS Collaboration is currently working on a concept for this new internal detector, which will consist entirely of silicon semi-conductor detectors. The innermost region is formed from a plurality of layers of pixel detectors, and strip detectors follow further outwards Fig. 2. Though to a different extent, both detectors have an extremely high power density of the readout electronics, which requires a correspondingly effective cooling (cooling power of $240 \mathrm{~kW})$.

From Fig. 1, the requirement non-ionizing energy loss (NIEL) value is $4 \times 10^{15}$ particles $/ \mathrm{cm}^{2}$. The NIEL value is a theoretical quantity, which makes it possible to easily compare different effects, which play a particular role in the damage of silicon detectors.

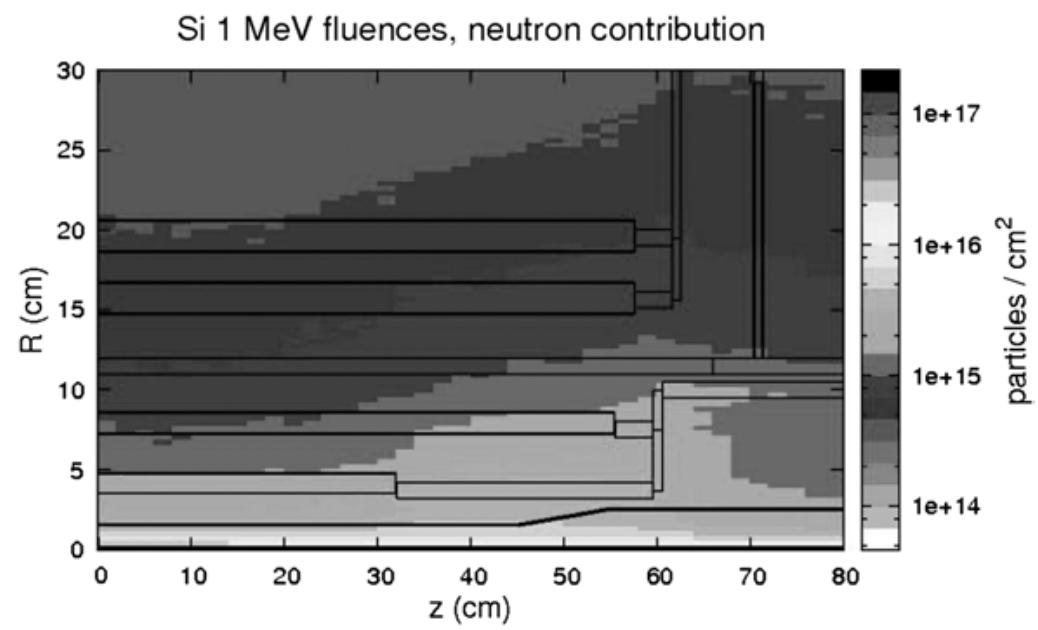

Fig. 1: The calculated non-ionizing energy loss. 


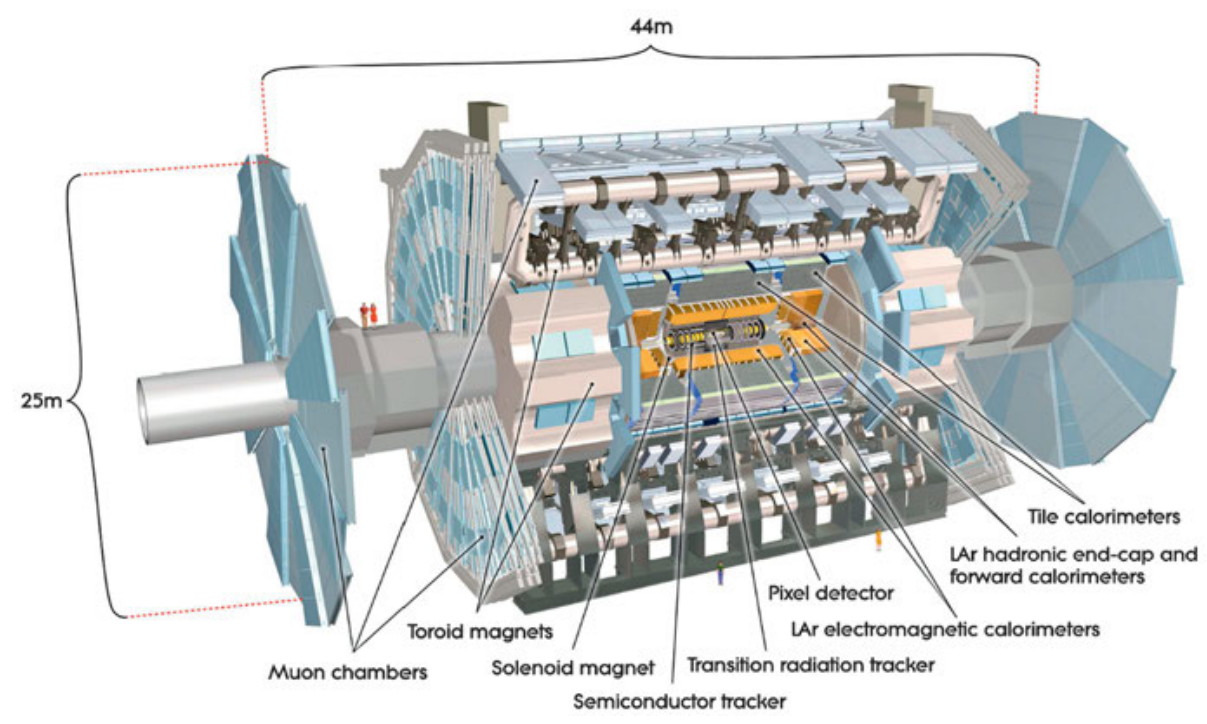

Fig. 2: The ATLAS Experiment at CERN.

An evaporative temperature of the coolant of $-40^{\circ} \mathrm{C}$ requires an extremely low $\mathrm{RH}$ of the atmosphere in order to avoid condensation on the electronics in all circumstances. Therefore, a permanent monitoring of the $\mathrm{RH}$ or the determination of the dew point by moisture sensors is an indispensable prerequisite for the safe operation of the detector (the ambient dew point should be $<-40^{\circ} \mathrm{C}$ ). In addition to measuring the very low amount of residual moisture at low temperatures, the radiation hardness of the moisture sensors is another requirement. Based on the fact that the ATLAS detector will be operating for 10 years on the HL-LHC, an integrated luminosity of $3000 \mathrm{fb}^{-1}$ is expected. The atmosphere will usually be steamed off nitrogen or highly filtered clean air without dust or oil residue, so that no contamination of the sensor by deposits is to be feared. In order to influence the radiation length as little as possible, the sensor should introduce little additional material into the detector volume or preferably consist of materials with a low mass number. For the same reason, the number of leads for supply and readout should be as low as possible. Due to the extreme packing density of the detector, the moisture sensor should also be as small as possible. Since ultimately the dew point is the critical variable, a temperature measurement should be integrated. The signal strengths must be sufficient to allow for transmission over at least 15-m cables. Depending on the type of the measured variable, local data processing is to be considered. The entire detector is located in a magnetic field of $2 \mathrm{~T}$.

The whole arrangement is designed for a duration of ten years. After the installation, there is no access capability, which consequently places the highest demands on the reliability of all installed components. Maximum stability of all calibration factors is necessary, unless the sensor system has a self-calibration. 


\subsection{Design of the radiation hard humidity sensor}

\subsubsection{Layout}

In order to achieve the required small size, silicon-on-insulator (SOI) material is used in order to be able to perform double-sided processing. This allows to use both the front and the rear of the chip for components. On the device side of the wafer with a thickness of $10 \mu \mathrm{m}$, the 3D inter-digital structure is produced by deep reactive ion etching (DRIE). The use of a 3D structure results in two advantages for the detector. First, the effective detector area is thereby increased with the same base area. Second, it is advantageous to use highly doped silicon instead of metal for the conductive structures. This is in accordance with the requirement to use materials with a small number of nuclear charges. This reduces the activation of the material by the irradiation and the radiation length is influenced as little as possible by the sensor.

Three different variants have been implemented with regard to the geometric layout. The combinations listed in Fig. 3 are used for the finger widths and distances between the fingers.

\begin{tabular}{rrr}
\hline gap width $[\mu \mathrm{m}]$ & gap depth $[\mu \mathrm{m}]$ & finger width $[\mu \mathrm{m}]$ \\
\hline 1.1 & 10 & 3.5 \\
1.5 & 10 & 2.5 \\
3.0 & 10 & 4.0 \\
\hline
\end{tabular}

The smaller the distance between the fingers, the higher the total capacitance of the sensor. On the other hand, small finger distances make it difficult to insert the moisture-sensitive material between the fingers. Since this is an RH sensor, the temperature must be measured simultaneously to determine the dew point temperature. This is done according to the formula

$$
T_{T}=f_{R}^{\frac{1}{8.02}}(109.8+T)-109.8
$$

where $T_{T}$ is the dew temperature point and $f_{R}$ is the $\mathrm{RH}$.

Like all other components, the diode, used to measure the temperature, is placed on the handle side of the wafer. In the first design, a micro-controller is used, which has very small geometric dimensions [3]. This concept is followed because the sensor must first be as small as possible, but on the other hand the output signals should be given via a 16-m long line (Fig. 4). In the case of the inter-digital structure, a square pulse is applied to the structure via a fixed resistor. On the basis of the capacitance, which has been adjusted due to the humidity, there is a decay time due to the RC constant.

This is analyzed by measuring the voltage after a fixed time $t$. In order to provide the information of this analog signal via the signal line, it is digitized with an ADC integrated on the micro-controller. The micro-controller used is commercial standardware 


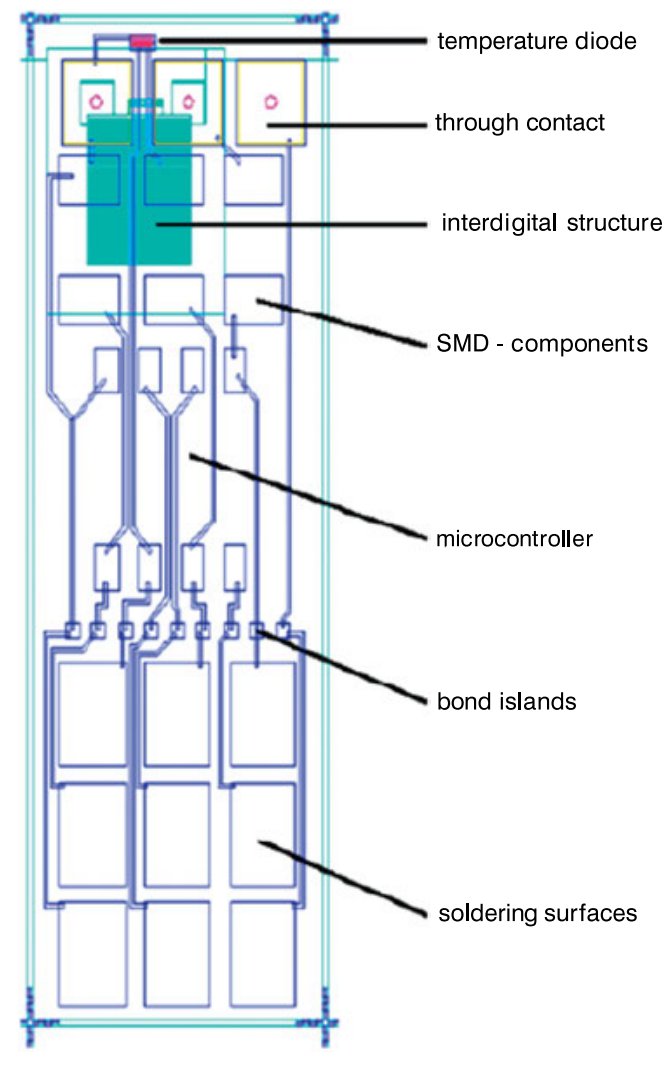

Fig. 3: Layout of the sensor.
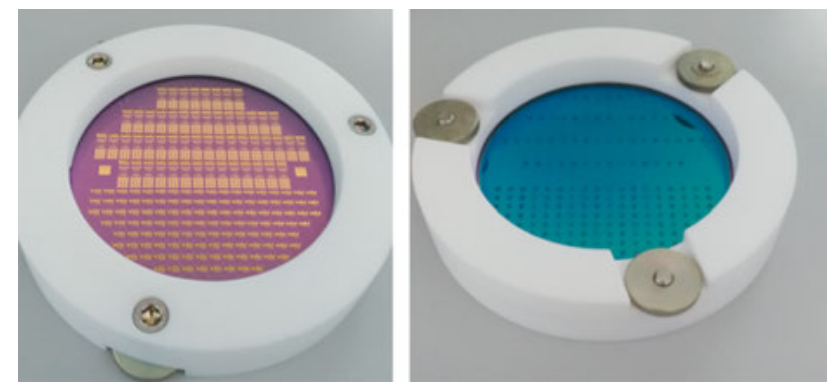

Fig. 4: Double-sided preparation of the wafer.

and therefore does not meet the increased requirements at CERN, in particular the very high radiation load. Therefore, this part is only a conceptual design and should be transferred from a sub-center of CERN to a layout with radiation-proof electronics. The large solder pads in the layout are only for programming the micro-controller and do not belong to the definitive concept of the sensor. For the online calibration of the system, the installation option of a capacitor is also provided as a reference on the circuit board. 
All previous RH sensors used in CERN failed due to their insufficient radiation resistance. The moisture-sensitive layer consists of polymer chains on all commercially available sensors. These are split at high beam exposures. In this way, the sensor changes its response behavior and thus its characteristics. In the concept pursued at $\mathrm{CiS}$, SOL-GELs are used. After processing, these form an $\mathrm{SiO}_{2}$ matrix in which some water binding substances are additionally incorporated. Furthermore, in the new development of the SOL-GEL used, no organic solvents were used but only water [4].

In dip coating, a sample is placed in a liquid medium to coat it. The sample is dipped into the substance, then remains there for a certain time $t$ and is then pulled out at a constant velocity $v_{o}$. After this, the sample has to dry for a certain time and may have to be dried again in an oven at higher temperatures. For this purpose, the layer thickness can be calculated using the Landau-Levich, i. e.,

$$
d=0.8 \sqrt{\frac{\eta v_{0}}{\rho g}} .
$$

The layer thickness $d$ is determined by this formula from the viscosity $\eta$, the drawing speed $v_{0}$, the density $\rho$ of the liquid and the gravitational constant $g$ [5].

For applying the SOL-GEL to the sensor layer, the spin coating method was unsuitable since the 3D sensor structures were not filled (Fig. 5). The best results were obtained by the dip coating method, whereby the structure to be coated is slowly drawn out of the solution and subsequently annealed. It was also found to be advantageous during the immersion phase to sonicate the solution with ultrasound in order to achieve a true homogeneous coating.
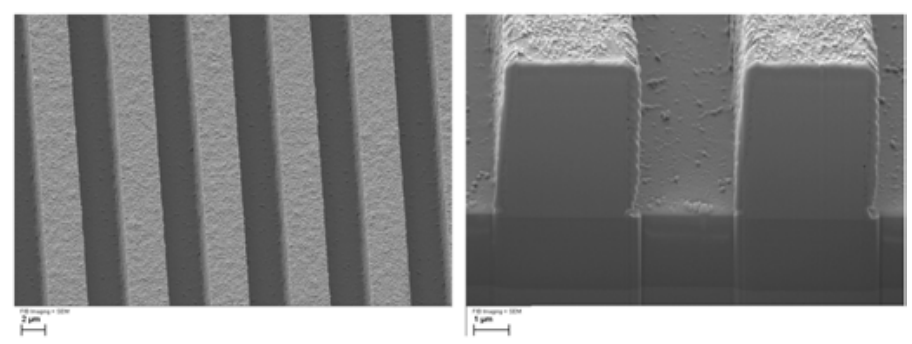

Fig. 5: 3D structure.

\subsection{Measurements}

\subsubsection{Uncoated sensors}

The sensors were initially measured dry and with de-ionized water using an LCR meter (Agilent 4294A). The results are shown in Fig. 6.

Significant differences of the sensor signal at room temperature in the dry state and with the applied drop of de-ionized water can be seen. The water quantity 


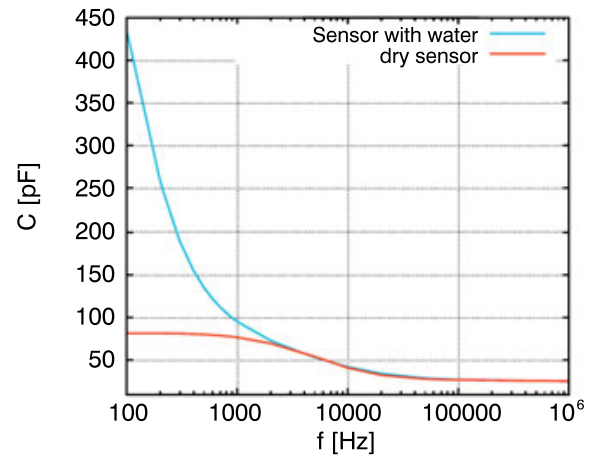

(a)

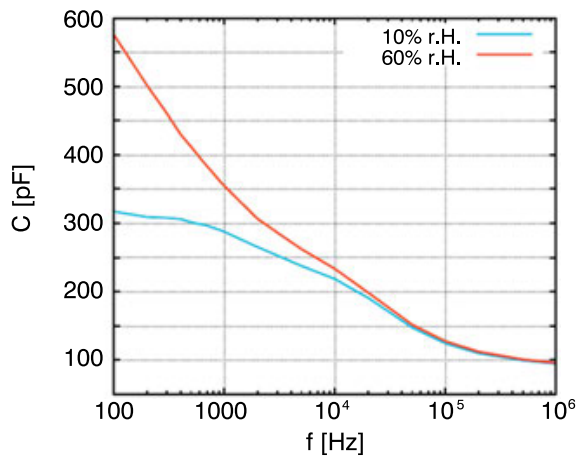

(b)

Fig. 6: (a) Uncoated Sensor. (b) Coated Sensor.

was $5 \mu \mathrm{L}$. The difference corresponds to about $350 \mathrm{pF}$ at $100 \mathrm{~Hz}$ and is thus easily detectable. In this way, the general functionality of the first built-in sensors was checked. After this function test, the sensors were provided with the moisture-sensitive layer for further measurements.

\subsubsection{Coating with dip coating}

The temperature was kept constant at $25^{\circ} \mathrm{C}$ during the measurements and the RH varied between $10 \%$ and $60 \%$. First, the capacitance was measured as a function of the frequency. The measured frequency range was between $100 \mathrm{~Hz}$ and $1 \mathrm{MHz}$. Fig. 7 shows the difference between the two moisture values.

Based on these measurements, a value of $5 \mathrm{pF} / \% \mathrm{RH}$ at $100 \mathrm{~Hz}$ can be calculated. The capacity at $100 \mathrm{~Hz}$ and $10 \% \mathrm{RH}$ is $317 \mathrm{pF}$ and with a humidity of $60 \% 575 \mathrm{pF}$ is

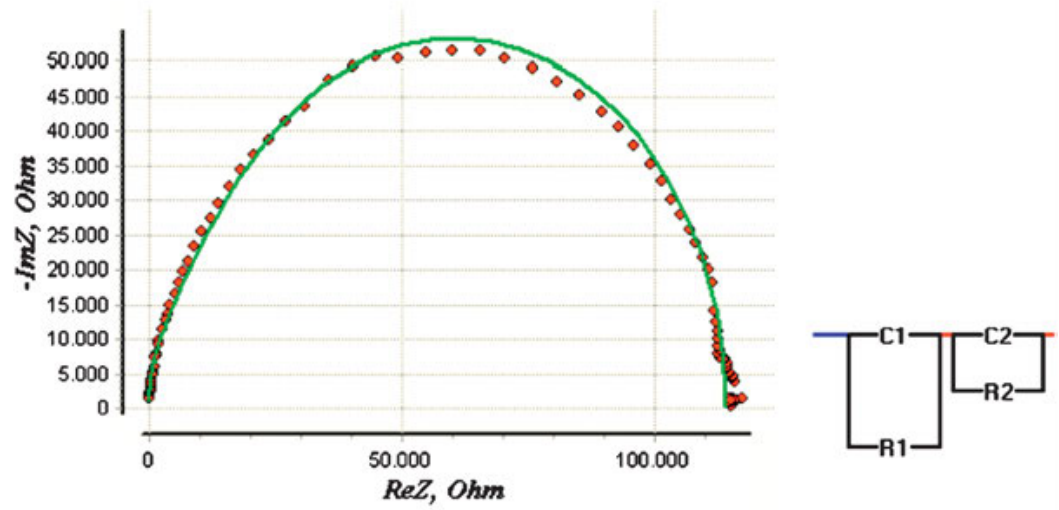

Fig. 7: Equivalent circuit. 
achieved. In addition, the impedance and the phase were measured in a frequency range of $25 \mathrm{~Hz}$ to $1 \mathrm{MHz}$. Again in this case, the difference between the different moisture values can be clearly seen. At $10 \% \mathrm{RH}$, the impedance is $6.89 \mathrm{MOhm}$ and with a humidity of $60 \% 3.71 \mathrm{MOhm}$. The course of the measurement curves corresponds to that of RC elements.

\subsubsection{Irradiation of samples}

To investigate whether the new layer used will withstand future radiation exposure without changing its required properties, several samples were irradiated at the 27-MeV proton linear accelerator in Birmingham [6]. Prior to irradiation and after irradiation, FTIR spectra were recorded to investigate whether the chemical bonding structures detectably altered. In order to obtain a better indication of the stability of the coating, different samples were irradiated at different doses. These had the values 0.77 MGy, 1.11 MGy, 4.84 MGy and 6.12 MGy. Even at the highest dose, no significant differences were found between irradiated and unirradiated specimens. In a next step, a mounted unit under irradiation is to be subjected to a functional test.

\section{Summary}

The experimental results so far suggest that the concept underlying the sensor works reasonably. The vias proved to be critical; the technology still requires a modification to make it less susceptible. Moreover, it was found that the method of dip coating for applying the sensitive layer appears to be the only practicable method. For this purpose, the process parameters such as pulling speed and tempering times had to be found by means of corresponding test series. Further, an additional application of ultrasound during the coating process was useful to achieve a more homogeneous coating image. Experiments with spin coating showed unsatisfactory results because the 3D structures are not filled sufficiently.

\section{Bibliography}

[1] CERN Document server, https://home.cern/about/experiments/atlas.

[2] Honeywell, HIH-4000 Series, https://sensing.honeywell.com.

[3] Flash-Based 8-Bit CMOS Microcontrollers PIC12F617 8-Pin, http://www.microchip.com/.

[4] N. Winkler, “GmBU Gesellschaft zur Förderung von Medizin”, Bio- und Umwelttechnologien.

[5] I. Buchem, "Nanostrukturierte Aluminiumfluoridschichten, Über das neuartige Niedertemperatur Sol-Gel Verfahren und die charakteristischen Eigenschaften", Dissertation Verlag; Dezember 2010.

[6] AIDA2020, "Advanced European Infrastructures for Detectors at Accelerators". 